\title{
Optical and chemical properties of tholins
}

\author{
Bishun N. Khare ${ }^{1}$, Christopher P. McKay ${ }^{2}$, Dale P. Cruikshank ${ }^{2}$, \\ Yasuhito Sekine $^{3}$, Patrick Wilhite ${ }^{4}$, and Tomoko Ishihara ${ }^{1}$ \\ ${ }^{1}$ Carl Sagan Center, SETI Institute, Space Science Division, NASA Ames Research Center, \\ USA \\ email: bkhare@mail.arc.nasa.gov \\ ${ }^{2}$ Space Science Division, NASA Ames Research Center \\ ${ }^{3}$ Department of Earth and Planetary Science, University of Tokyo, 7-3-1 Hongo, Bunkyo, \\ Tokyo 113-0033, Japan \\ ${ }^{4}$ Center for Nanostructures, School of Engineering, Santa Clara University, Santa Clara \\ California 95053-0569
}

\begin{abstract}
For over three decades tholins have been synthesized from mixtures of the cosmically abundant gases $\mathrm{CH}_{4}, \mathrm{C}_{2} \mathrm{H}_{6}, \mathrm{NH}_{3}, \mathrm{H}_{2} \mathrm{O}, \mathrm{HCHO}, \mathrm{N}_{2}$, and $\mathrm{H}_{2}$, previously in the Laboratory for Planetary Studies at Cornell University and in recent years at NASA Ames Research Center. The tholin synthesized by UV light or spark discharge on sequential and non-sequential pyrolysis GC-MS revealed hundreds of compounds, and on hydrolysis produced a large number of amino acids including racemic protein amino acids. Optical constants have been measured of many of the tholins, tholins produced from a condensed mixture of water and ethane at $77 \mathrm{~K}$, poly $\mathrm{HCN}$, and Titan tholin produced on electrical discharge through a mixture of $90 \% \mathrm{~N}_{2}$ and $10 \% \mathrm{CH}_{4}$. Its optical constants were measured from soft x-rays to microwave for the first time.

Here we report the absorption properties of Titan tholin that is produced in the temperature range 135 to $178 \mathrm{~K}$ where tholins are produced by magnetospheric charged particles, then pass through lower temperature at $70 \mathrm{~K}$ and finally to the ground at $95 \mathrm{~K}$. While descending to the ground, it gets coated and processed on the way by other sources of energy such as long UV and cosmic rays. It is therefore expected that the stable products of $\mathrm{CH}_{4}$ photolysis react with Titan tholin to recycle the $\mathrm{CH}_{4}$ supply in Titan's atmosphere. Furthermore, the reactions of gaseous $\mathrm{C}_{2} \mathrm{H}_{6}$ with the reactive materials on the surface of the tholin could incorporate atmospheric $\mathrm{C}_{2} \mathrm{H}_{6}$ into the tholin and therefore might reduce the deposition rate of $\mathrm{C}_{2} \mathrm{H}_{6}$ onto the ground of Titan.
\end{abstract}

\section{Discussion}

LORENZ: You suggest that the carbon in tholin might be recycled into the atmosphere as methane but I don't understand how you can recycle methane if the hydrogen escapes to space. The hydrogen budget doesn't close. Can you explain how you get around that?

KHARE: Well there is some evidence that hydrogen is absorbed as well, but I am still trying to confirm this using deuterium.

LoRENZ: But on the planetary scale hydrogen is escaping from Titan.

KHARE: There is a possibility that some hydrogen may be absorbed on the tholin. If tholin could behave like a chromatographic column, it could absorb species and release them as a gas when it absorbs energy. In Titan's case, you have all kinds of energy falling on the tholin at various heights. It is very important to consider the concept that the real Titan tholin is likely coated with materials as it descends to the ground. The tholin has absorption characteristics and maybe catalytic properties too. Our job now is to create 
tholin under Titan temperatures and pressures and then examine its properties without exposing it to air.

LORENZ: I still maintain that, whatever exchanges and reactions occur, on the planetary scale you cannot solve the methane survival problem with tholin.

KHARE: Our current research tells us that tholin can be at least a partial contributor to the available methane for astronomical periods of time with the same mixing ratio.

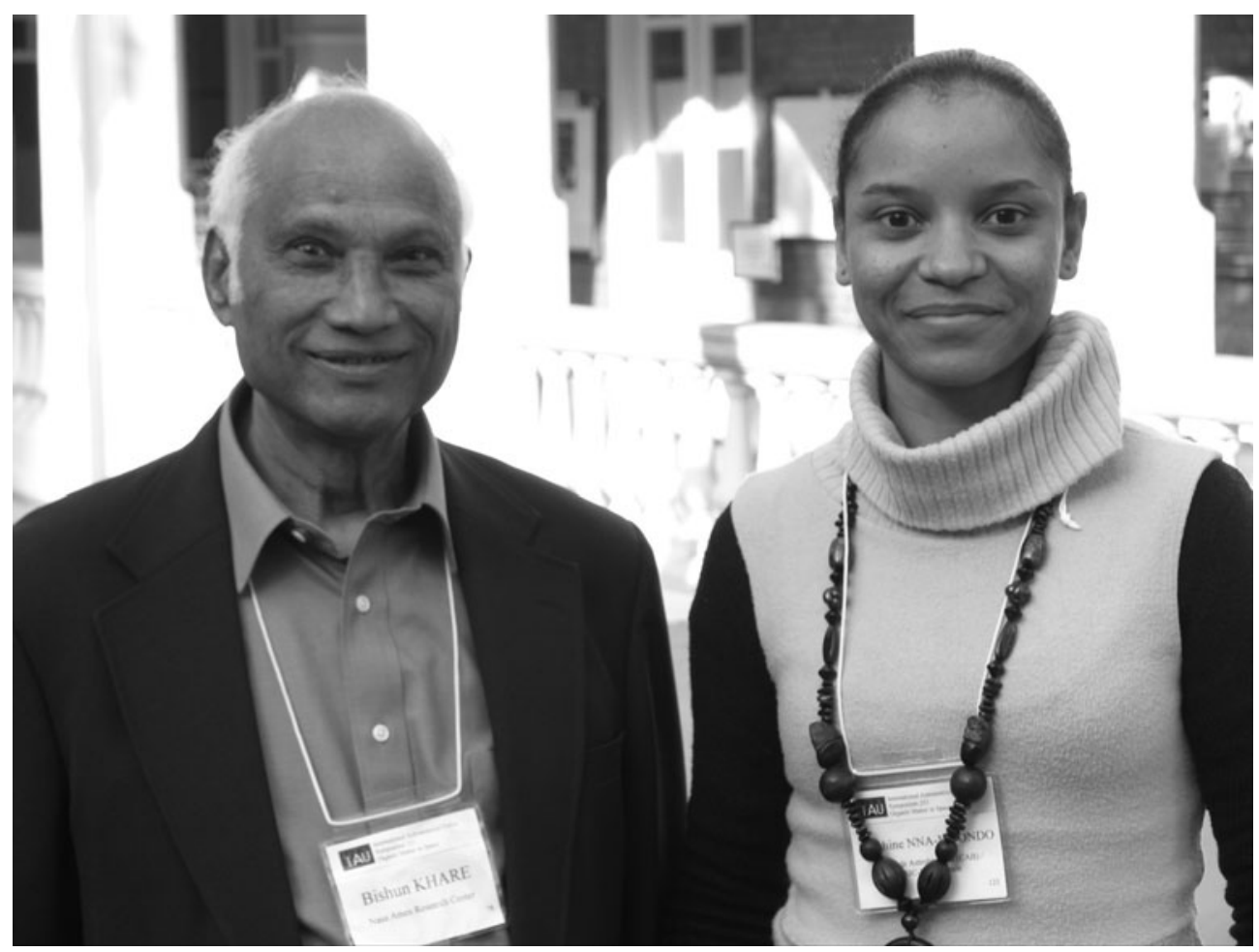

Bishun Khare and Delphine Nna-Mvondo (photo by Dale Cruikshank) 